\title{
GW23-e1066 EFFECT OF CELL-CELL DIRECT CONTACT TO MESENCHYMAL STEM CELLS DIFFERENTIATE INTO VASCULAR ENDOTHELIAL CELLS
}

doi:10.1136/heartjnl-2012-302920a.269

Jing Peng, Luxiang Chi. Department of Cardiology, Southwest Hospital, Third Military Medical University, Chongqing

Objectives To investigate the effect of cell-cell direct contact to mesenchymal stem cells (MSCs) differentiate into vascular endothelial cells (VECs) when MSCs co-cultured with human umbilical vein endothelial cells (HUVECs) in vitro.

Methods MSCs and HUVECs were cultured and identified, and then co-culture. The VEGF levels of experimental group (culture media of co-cultured for $48 \mathrm{~h}$ ) and control group (MSCs and HUVECs's mixed culture media after them were cultured alone for $48 \mathrm{~h}$ ) were confirmed using ELISA assays. Co-cultured for 5 days, The MSCs's expression of fetal liver kinase-1 (Flk-1) and von Willebrand factor (vWF) and ingestible ability of Dil-ac-LDL was analysed by immunofluorescence staining, and the changes of ultrastructure of co-cultured cells were observed.

Results The VEGF levels of experimental group was significantly higher than control group; In direct contact co-culture system, some MSCs were induced to express Flk-1 protein, and part of the Flk-1 positive MSCs also expressed vWF protein ;some MSCs could phagocytose Dil-ac-LDL; the reduced karyoplasmic ratio and abundant organelles were observed in the co-cultured MSCs by transmission electron microscopy, and cell fusion can be seen.

Conclusions UVECs induce MSCs to differentiate into endothelial cells through direct cell contact by direct co-culture, the mechanism may be closely related to the formation of gap junction and the promotion of VEGF secretion by in direct contact and cell fusion. 